\title{
Potentiation of Pentazocine Analgesia by Low-dose Naloxone
}

Jon D. Levine, ${ }^{\star \star}$ Newton C. Gordon, ${ }^{\ddagger}$ Yetunde O. Taiwo, ${ }^{\ddagger}$ and Terence J. Coderre ${ }^{\ddagger}$

*Departments of Medicine and ${ }^{\ddagger}$ Oral and Maxillofacial Surgery, Schools of Medicine and Dentistry, University of California, San Francisco, San Francisco, California 94143

\section{Abstract}

The analgesia produced by combinations of low-dose naloxone with pentazocine or morphine was studied in 105 patients with moderately severe postoperative pain after standardized surgery for removal of impacted third molars. Pain intensity was quantified using a visual-analogue scale. To eliminate the release of endogenous opioids produced by the placebo component of open drug administration, all injections were made by a preprogrammed infusion pump. The analgesia produced by pentazocine, an agonist-antagonist opiate-analgesic acting predominantly at the kappa opiate receptor, was potentiated by low-dose naloxone, whereas the analgesia produced by morphine, a mu-agonist, was attenuated by low-dose naloxone. To evaluate whether similar potentiation would be present in an animal model, and specifically, in the absence of diazepam, which patients receive, we performed an analogous experiment in rats in which nociceptive threshold was determined using the Randall-Selitto paw-withdrawal test. The results were completely analogous to the clinical results: pentazocine analgesia was potentiated by low-dose naloxone, whereas morphine analgesia was attenuated by low-dose naloxone. These data demonstrate a novel interaction between opiates, and suggest a rationale for opiate combinations to produce potent analgesia with fewer autonomic side effects and less abuse potential than presently available analgesics.

\section{Introduction}

The opiate-antagonist naloxone, administered at high doses, increases pain when administered in the presence of opiateanalgesics or under conditions when endorphins are probably released (1-4). When administered at low doses, however, naloxone produces analgesia in humans (3-5) and raises nociceptive thresholds in animals (6-8). Although of low potency, naloxone has valuable features that would make it an "ideal" analgesic. It does not produce the common side effects that are present with other opiate analgesics (mental obtundation, cardiovascular and respiratory depression, constipation, and dysphoria) (9), and it has low abuse and addiction potential (9).

Since it is stereospecific, the increase in pain produced by naloxone is thought to be due to its antagonism of endogenous opioid-peptide interactions with opiate receptors (10). The

Address correspondence to Dr. Jon D. Levine, Division of Rheumatology and Clinical Immunology, U-426 (Box 0724), University of California, San Francisco, CA 94143-0724.

Received for publication 18 June 1987 and in revised form 10 May 1988.

J. Clin. Invest.

(C) The American Society for Clinical Investigation, Inc.

$0021-9738 / 88 / 11 / 1574 / 04 \$ 2.00$

Volume 82, November 1988, 1574-1577 mechanism underlying low-dose naloxone analgesia, however, is unknown. That low-dose naloxone produces analgesia by an unusual mechanism is suggested by the fact that the biphasic dose-dependent relationship of its effect on pain does not resemble the dose-dependent relationship of any other opiate analgesic; i.e., no other opiate produces analgesia only at low doses.

Opiate agonists produce analgesia by acting at one or more receptors (i.e., mu, kappa, and delta) (11-13). A single opiate administered simultaneously at different sites in the central nervous system can synergize to produce greater analgesia (14, 15). Since naloxone is believed to interact with opiate receptors (i.e., it produces hyperalgesia at high doses) we hypothesized that low-dose naloxone might be able to potentiate the analgesia of other opiates. We report that the analgesia produced by pentazocine, an agonist-antagonist opiate-analgesic acting predominantly at the kappa opiate receptor $(16,17)$, is potentiated by low-dose naloxone, whereas the analgesia produced by morphine, an agonist opiate-analgesic at the mu opiate receptor, is attenuated by low-dose naloxone. Furthermore, we report that this interaction exists in a standard animal model.

\section{Methods}

105 patients underwent standardized surgery for the removal of impacted third molars after premedication with intravenous diazepam. The surgical procedure and the visual-analogue scale used for measuring pain have been described in detail elsewhere (18). During surgery, nitrous oxide and local anesthesia (carbocaine without vasoconstrictor) were used. The duration of the surgery and experiment, measured from the onset of local anesthesia, was $\sim 5 \mathrm{~h}$.

After surgery, each patient was randomly assigned to receive, via an indwelling intravenous line, a double-blind injection of either vehicle, $0.4 \mathrm{mg}$ low-dose naloxone, 8 or $15 \mathrm{mg}$ morphine, $60 \mathrm{mg}$ pentazocine, $0.4 \mathrm{mg}$ low-dose naloxone with $8 \mathrm{mg}$ morphine, or $0.4 \mathrm{mg}$ low-dose naloxone with $60 \mathrm{mg}$ pentazocine. All injections were made by a preprogrammed infusion pump. This method of drug administration eliminates the release of endogenous opioids produced by the placebo component of open drug administration (15). The time of substance administration was varied randomly between $1 \mathrm{~h}$ and $20 \mathrm{~min}$ and $2 \mathrm{~h}$ (average, $1 \mathrm{~h}$ and $40 \mathrm{~min}$ ) after the onset of anesthesia and was the same for all groups of patients.

Since baseline pain levels (Fig. 1) did not differ significantly among the seven experimental groups $(F[6,98]=0.6$, NS), the magnitude of the analgesic effect of each intervention was defined as the change from the pain intensity before drug administration to $50 \mathrm{~min}$ (peak analgesia) and to $3 \mathrm{~h}$ and $10 \mathrm{~min}$ (end of experiments after administration of a drug or combination of two drugs). Magnitudes of analgesic effect for the various drug treatments were compared using Neuman-Keuls' post hoc comparison (19) after analysis of variance, which demonstrated significant difference between the groups both at $50 \mathrm{~min}(F[1,6]$ $=10.8, P<0.0001)$ and at $3 \mathrm{~h}$ and $10 \mathrm{~min}(\mathrm{~F}(1,6)=4.0, P<0.01)$.

Mechanical nociceptive thresholds were measured in 250-300-g male Sprague-Dawley rats (Bantin \& Kingman, Fremont, CA) using a Basile analgesymeter (Stoelting Co., Chicago, IL), which generates a 


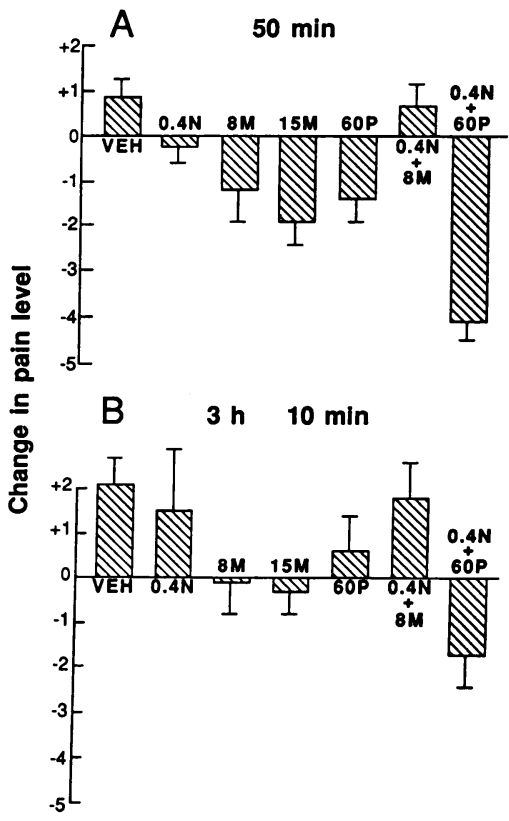

or $0.4 \mathrm{mg}$ naloxone and $60 \mathrm{mg}$ pentazocine $(0.4 \mathrm{~N}+P)$, compared with pretreatment baseline. Drugs were always administered midway between two pain ratings. Pretreatment baseline pain intensities were: VEH (5.2), 0.4N (5.0), 8M (4.7), 15M (4.5), 60P (4.4), $0.4 \mathrm{~N}$ $+8 \mathrm{M}(4.6)$, and $0.4 \mathrm{~N}+60 \mathrm{P}(5.3)$.

linearly increasing mechanical force that is applied by a conical piece of plastic with a dome-shaped tip, on the dorsal surface of the rat's hindpaw $(20,21)$. The nociceptive threshold is defined as the force in grams at which the rat withdraws its paw. The rats were trained in this procedure for $2 \mathrm{~h}$ daily in the week before experimentation. On the day of the experiment, the rats were exposed to the test stimulus at 5-min intervals for $2 \mathrm{~h}$ before the measurement of baseline thresholds. The baseline threshold was defined as the mean of the last six determinations. Rats were then injected with graded subcutaneous doses of opioid, or opioid combinations. Vehicle injection has been shown to have no effect $(20,21)$. The nociceptive thresholds were remeasured 20 min after this injection. Since baseline nociceptive thresholds did not differ significantly among the seven experimental groups $(F[6,110]$ $=1.75$, NS), the magnitude of the analgesic effect of each intervention was defined as the percentage change from the pain intensity before drug administration to $20 \mathrm{~min}$ (the time of peak analgesia) after administration of the drug or drug combination. Post hoc comparisons of the analgesic effects of the various treatments were based on a multiple stage $F$ test (22) after an analysis of variance that demonstrated a significant difference between the groups $(F[6,110]=6.4, P<0.0001)$.

\section{Results}

Clinical studies. The mean pain intensity, measured $50 \mathrm{~min}$ after injections, was increased in the group receiving vehicle (Fig. $1 A$ ). Compared with this control group, mean pain intensity was decreased in groups that received either morphine ( 8 and $15 \mathrm{mg})(P<0.05$ and $P<0.01$, respectively) or pentazocine $(60 \mathrm{mg} ; P<0.05)$ as a single agent. To study the interaction between low-dose naloxone and the two opiate analgesics, the groups receiving low-dose naloxone combined with either morphine or pentazocine were compared with the groups receiving each member of the combination administered alone, as well as to the group receiving high-dose (15 $\mathrm{mg})$ morphine. The combination of low-dose naloxone and penta- zocine produced significantly greater analgesia than either low-dose naloxone $(P<0.01)$, pentazocine $(P<0.01)$, or even high-dose morphine administered alone $(P<0.01)$. The combination of low-dose naloxone and $8 \mathrm{mg}$ morphine, however, produced less analgesia when compared with the same dose of morphine alone $(P<0.05)$ or with high-dose morphine $(P$ $<0.01)$ but not when compared with low-dose naloxone administered alone.

Compared with the control group, the mean pain intensity measured at $3 \mathrm{~h}$ and $10 \mathrm{~min}$ after injection of single analgesic agents was not significantly decreased (Fig. $1 \mathrm{~B}$ ). In addition, the analgesia produced by the combination of low-dose naloxone and $8 \mathrm{mg}$ morphine did not differ significantly from the analgesia produced by the same dose of morphine. The combination of low-dose naloxone and pentazocine, however, continued to produce significant analgesia when compared with either agent alone (both $P<0.01$ ). That is, by $3 \mathrm{~h}$ and $10 \mathrm{~min}$ after injection only the group of patients receiving low-dose naloxone plus pentazocine still reported significant analgesia.

Animal studies. To address the possibility, raised during the review process, that an interaction with diazepam was responsible for the results, we performed an analogous experiment in rats in which diazepam could be omitted.

Naloxone produced a biphasic dose-dependent analgesia. At low doses (50 ng/kg, $500 \mathrm{ng} / \mathrm{kg}$, and $5 \mu \mathrm{g} / \mathrm{kg}$ ) naloxone produced analgesia; this disappeared when naloxone doses were increased to $50 \mu \mathrm{g} / \mathrm{kg}$ and $500 \mu \mathrm{g} / \mathrm{kg}$ (Fig. 2 A). Pentazocine and morphine both produced dose-dependent analgesia (Fig. 2, $B$ and $C$ ).

When administered in combinations, $50 \mathrm{ng}$ naloxone, which by itself produced a $9.2 \pm 3.5 \%(n=9)$ increase in nociceptive threshold, significantly reduced the analgesia produced by $1 \mathrm{mg}$ morphine (from $34.7 \pm 12.6 \%(n=11)$ to $13.8 \pm 2.9 \%(n$ $=19 ; P<0.05)$ (Fig. 3). On the other hand, the same dose of naloxone, when given in combination with a $5-\mu \mathrm{g}$ dose of pentazocine, which by itself produces an increase in nociceptive threshold of $24.8 \pm 3.0 \%(n=23)$, produces a resultant increase in threshold of $53.1 \pm 7.9 \%(n=10)$. This is significantly greater than either agent alone $(P<0.01$ and $P<0.01$, respectively) and even greater than the effect of a 10 -fold-
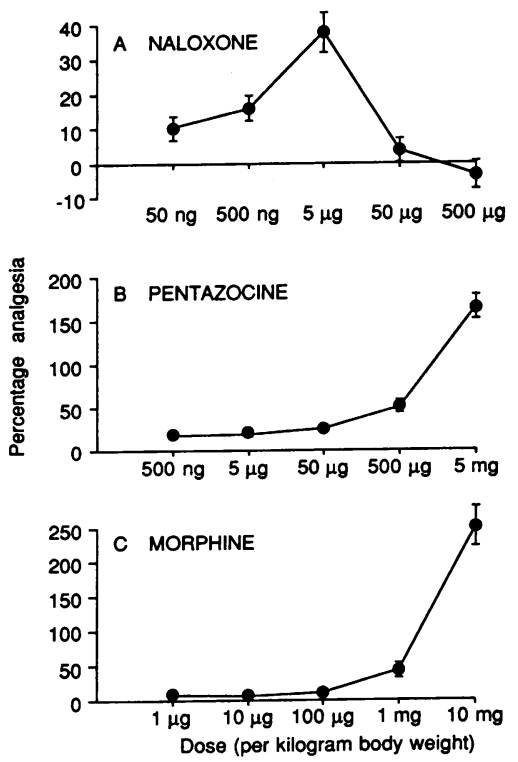

Figure 2. Dose dependence of effects of subcutaneous naloxone $(A)$, pentazocine $(B)$, and morphine $(C)$ on the paw-withdrawal threshold. Responses are graphed as percentage change from baseline after administration of the various agents. Each point in the figure represents the mean \pm 1 SEM of $n=22$ (naloxone), $n=36$ (pentazocine), and $n=18$ (morphine) rats. 


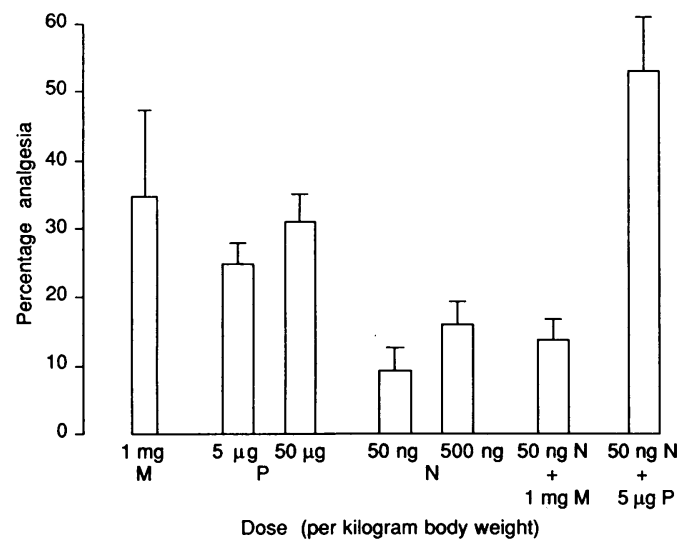

Figure 3. The effects of $1 \mathrm{mg}$ s.c. morphine $(M)(n=11)$, pentazocine $(P)(5 \mu \mathrm{g}, n=23 ; 50 \mu \mathrm{g}, n=23)$, and naloxone $(N)(50 \mathrm{ng}, n$ $=9 ; 500 \mathrm{ng}, n=22$ ) alone, and combinations of $50 \mathrm{ng}$ naloxone with $1 \mathrm{mg}$ morphine $(n=19)$, and $5 \mu \mathrm{g}$ pentazocine with $50 \mathrm{ng}$ naloxone $(n=10)$ on paw withdrawal threshold. Responses are graphed as \% change from baseline to $20 \mathrm{~min}$ after the administration of various agents. All statistical tests were performed on change scores using a multiple stage $F$-test following analysis of variance. Each bar represents the mean \pm 1 SEM of a particular group.

higher dose of pentazocine ( $50 \mu \mathrm{g}, 30.9 \pm 4.1, n=23, P<0.05)$ or naloxone $(500 \mathrm{ng}, 15.9 \pm 3.5 \%, n=22, P<0.01)$.

\section{Discussion}

In this study, we have evaluated the analgesic effect resulting from the administration of a low dose of the opiate-antagonist naloxone along with two opiate agonists, morphine (a mu type) and pentazocine (a kappa type), to patients with postoperative pain and to animals during nociceptive threshold testing. Since naloxone is a potent antagonist of the mu-type opiate receptor, at which morphine acts to produce analgesia, the observed antagonism of morphine analgesia by naloxone was expected. However, pentazocine and low-dose naloxone unpredictably produced a more potent and prolonged analgesia than either drug alone. This analgesia was, in fact, significantly greater than that produced by high-dose morphine, even though kappa-agonists such as pentazocine are less potent than mu-agonists such as morphine, and produce analgesia of shorter duration $(23,24)$. Since the half-life of naloxone in humans is only $60 \mathrm{~min}(25)$, the prolonged (up to $3 \mathrm{~h}$ ) analgesia produced by low-dose naloxone plus pentazocine in humans suggests that significantly lower doses of naloxone than those employed in this study may be sufficient to potentiate pentazocine analgesia. The finding of a marked potentiation of pentazocine analgesia by low-dose naloxone is clinically significant. This combination might allow adequate pain control with fewer autonomic side effects and abuse potential than occurs with presently available treatments.

Patients received intravenous diazepam at the time of surgery. Although there appeared to be no residual sedative effect at the time of analgesic administration ( $\sim 2 \mathrm{~h}$ later), an interaction with diazepam could not be excluded as a confounding factor in the synergy between pentazocine and low-dose naloxone, due to its long pharmacological half-life. For ethical reasons our dental protocol requires the use of a preoperative sedative. Animal studies were therefore performed to address this issue. Potentiation of pentazocine analgesia and antagonism of morphine analgesia by low-dose naloxone was seen in awake restrained rats who received no diazepam. A comparable synergy in another animal model has also been recently reported for naloxone and another agonist-antagonist, buprenorphine (26). It is reasonable, therefore, to assume that our clinical results were not due to an interaction with diazepam.

Although potent opiate synergism has been described previously, e.g., when morphine has been simultaneously injected into both the intrathecal and intracerebroventricular spaces $(14,15)$, the synergism that has been reported for combinations of opiate agonists and antagonists (26) is not well understood. A possible explanation for the potentiation of pentazocine analgesia by low-dose naloxone would be the presence of positive cooperativity between specific binding sites for naloxone and pentazocine. There is a precedent for this. Positive cooperativity has been demonstrated for interactions between two binding sites for the same opiate $(27,28)$ for mu and delta binding sites in vitro (29) and for mu- and delta-mediated analgesia in vivo (30-32).

In summary, this study has demonstrated a marked analgesia, more potent than that of high-dose morphine, produced by the combination of low-dose naloxone and pentazocine. This finding is of considerable experimental as well as clinical importance since it demonstrates a novel, potent interaction between different opiates as well as a rationale for new opiate combinations that produce profound analgesia.

\section{Acknowledgments}

We are grateful to Drs. Allan Basbaum, Philip Heller, and Franklin Perry for statistical consultation and review of the manuscript.

This work was supported by National Institutes of Health grants DE05369, NS21642, and AM32634. Dr. Levine is a Rita Allen Foundation Fellow.

\section{References}

1. LeBars, D., G. Guilbaud, I. Jurna, and J. M. Besson. 1976. Differential effects of morphine on responses of dorsal horn lamina V-type cells elicited by $A$ and $C$ fibre stimulation in the spinal cat. Brain Res. 115:518-524.

2. Frederickson, R. C. A., V. Burgis, and J. D. Edwards. 1977. Hyperalgesia induced by naloxone follows diurnal rhythm in responsivity to painful stimuli. Science (Wash. DC). 198:756-758.

3. Levine, J. D., N. C. Gordon, and H. L. Fields. 1979. Naloxone dose-dependently produces analgesia and hyperalgesia in postoperative pain. Nature (Lond.). 278:740-741.

4. Martin, W. R. 1984. Pharmacology of opioids. Pharmacol. Rev. 53:283-323.

5. Buchsbaum, M. S., G. C. Davis, and W. E. Bunney. 1977. Naloxone alters pain perception and somatosensory evoked potentials in normal subjects. Nature (Lond.). 270:620-622.

6. Sewell, R. D. E., and P. S. J. Spencer. 1976. Antinociceptive activity of narcotic agonist and partial agonist analgesics and other agents in the tail-immersion test in mice and rats. Neuropharmacology. 15:683-688.

7. Woolf, C. J. 1980. Analgesia and hyperalgesia produced in the rat by intrathecal naloxone. Brain Res. 189:593-597.

8. Kayser, V., and G. Guilbaud. 1982. Dose-dependent analgesic and hyperalgesic effects of systemic naloxone in arthritic rats. Brain Res. 226:344-348.

9. Jasinski, D. R., W. Martin, and C. A. Haertzen. 1967. The 
human pharmacology and abuse potential of $\mathrm{N}$-allylnoroxymorphone (naloxone). J. Pharmacol. Exp. Ther. 157:420-426.

10. Jacob, J. J. C., and K. Ramabadran. 1978. Enhancement of a nociceptive reaction by opioid antagonists in mice. $\mathrm{Br}$. J. Pharmacol. 64:91-98.

11. Ward, S. J., and A. E. Takemori. 1983. Relative involvement of mu, kappa and delta receptor mechanisms in opiate-mediated antinociception in mice. J. Pharmacol. Exp. Ther. 224:525-530.

12. Porreca, F., H. I. Mosberg, R. Hurst, V. J. Hruby, and T. F. Burks. 1984. Roles of mu, delta and kappa opioid receptors in spinal and supraspinal mediation of gastrointestinal transit effects and hotplate analgesia in the mouse. J. Pharmacol. Exp. Ther. 230:341-348.

13. Schmauss, C., and T. L. Yaksh. 1984. In vivo studies on spinal opiate receptor systems mediating antinociception. II: pharmacological profiles suggesting a differential association of mu, delta and kappa receptors with visceral chemical and cutaneous thermal stimuli in the rat. J. Pharmacol. Exp. Ther. 228:1-12.

14. Yeung, J. C., and T. A. Rudy. 1980. Multiplicative interaction between narcotic agonisms expressed at spinal and supraspinal sites of antinociceptive action as revealed by concurrent intrathecal and intracerebroventricular injections of morphine. J. Pharmacol. Exp. Ther. 215:633-642.

15. Levine, J. D., S. R. Lane, N. C. Gordon, and H. L. Fields. 1982. A spinal opioid synapse mediates the interaction of spinal and brainstem sites in morphine analgesia. Brain Res. 236:85-91.

16. Gilbert, P. E., and W. R. Martin. 1976. The effects of morphine- and nalorphine-like drugs in the nondependent, morphine-dependent and cyclazocine-dependent chronic spinal dog. J. Pharmacol. Exp. Ther. 198:66-82.

17. Tyers, M. B. 1980. A classification of opiate receptors that mediate antinociception in animals. Br. J. Pharmacol. 69:503-512.

18. Levine, J. D., and N. C. Gordon. 1984. Influence of the method of drug administration on analgesic response. Nature (Lond.). 312:755-756.

19. Zar, J. H. 1984. Biostatistical Analysis. Prentice-Hall, Englewood Cliffs, NJ. Second ed.

20. Levine, J. D., Y. O. Taiwo, S. D. Collins, and J. K. Tam. 1986. Noradrenaline hyperalgesia is mediated through interaction with sym- pathetic postganglionic neurone terminals rather than activation of primary afferent nociceptors. Nature (Lond.). 323:158-160.

21. Levine, J. D., W. Lau, G. Kwiat. 1984. Leukotriene $B_{4}$ produces hyperalgesia that is dependent on polymorphonuclear leukocytes. Science (Wash. DC). 225:743-745.

22. Statistical Analysis Systems Institute, Cary, NC. 1982. SAS User's manual.

23. Beaver, W. T., S. L. Wallenstein, R. W. Houde, and A. Rogers. 1966. A comparison of the analgesic effects of pentazocine and morphine in patients with cancer. Clin. Pharmacol. Ther. 7:740-751.

24. Goldstein, G. 1985. Pentazocine. Drug Alcohol Depend. 14:313-324.

25. Ngai, S. H., B. A. Berkowitz, J. C. Yang, J. Hempstead, and S. Spector. 1976. Pharmacokinetics of naloxone in rats and in man. Anesthesiology. 44:398-401.

26. Bergman, S. A., R. L. Wynn, D. E. Myers, and F. G. Rudo. 1988. Low dose naloxone enhances buprenorphine in a tooth pulp antinociceptive assay. Arch. Int. Pharmacodyn. Ther. 291:229-237.

27. Hazum, E., K.-J. Chang, H. J. Leighton, O. W. Lever, Jr, and P. Cuatrecasas. 1982. Increased biological activity of dimers of oxymorphone and enkephalin: possible role of receptor crosslinking. Biochem. Biophys. Res. Commun. 104:347-353.

28. Coy, D. H., A. J. Kastin, M. J. Walker, R. F. McGivern, and C. A. Sandman. 1978. Increased analgesic activities of a fluorinated and a dimeric analogue of [D-ALA-2]-methionine enkephalinamide. Biochem. Biophys. Res. Commun. 83:977-983.

29. Rothman, R. B., and T. C. Westfall. 1982. Allosteric coupling between morphine and enkephalin receptors in vitro. Mol. Pharmacol. 21:548-557.

30. Larson, A. E., J. L. Vaught, and A. E. Takemori. 1980. The potentiation of spinal analgesia by leucine enkephalin. Eur. J. Pharmacol. 6:381-383.

31. Lee, N. M., L. F. Leybin, J. K. Chang, and H. H. Loh. 1980. Opiate and peptide interaction: effect of enkephalin on morphine analgesia. Eur. J. Pharmacol. 68:181-185.

32. Vaught, J. L., R. B. Rothman, and T. C. Westfall. 1982. Mu and delta receptors: their role in analgesia and in the differential effects of opioid peptides on analgesia. Life Sci. 30:1443-1455. 